\title{
Randomization in the First Hitting Time Problem*
}

\author{
Ken Jackson ${ }^{\dagger} \quad$ Alexander Kreinin ${ }^{\ddagger} \quad$ Wanhe Zhang $\$$
}

February 10, 2009

\begin{abstract}
In this paper we consider the following inverse problem for the first hitting time distribution: given a Wiener process with a random initial state, probability distribution, $F(t)$, and a linear boundary, $b(t)=\mu t$, find a distribution of the initial state such that the distribution of the first hitting time is $F(t)$. This problem has important applications in credit risk modeling where the process represents, so-called, distance to default of an obligor, the first hitting time represents a default event and the boundary separates the healthy states of the obligor from the default state. We show that randomization of the initial state of the process makes the problem analytically tractable.
\end{abstract}

Primary Subjects: $60 \mathrm{G} 40$

Secondary Subjects: $91 \mathrm{~B} 70$

Keywords: First hitting time; inverse boundary problem;

\section{Introduction}

Let $X_{t}$ be an arbitrary process with continuous sample paths and $b(t)$ be a continuous absorbing boundary, satisfying the relation $X_{0} \geq b(0)$. The random variable

$$
\tau= \begin{cases}\inf \left\{t \geq 0: X_{t}<b(t)\right\}, & \text { if there exists } t \text { such that } X_{t}<b(t), \\ \infty, & \text { otherwise, }\end{cases}
$$

is called the first hitting time for the process $X_{t}$.

If $X_{t}$ is a diffusion process, the problem of finding the distribution of $\tau$ is a classical one; it was solved by A. Khintchine in [11] for sufficiently smooth boundaries. The solution to this problem was expressed in terms of a boundary value problem for the associated partial differential operator. Since then many books and research papers were published in this area. The monograph [12] summarizes known analytical results obtained by the mid-1980s, in [10]

\footnotetext{
${ }^{*}$ This research was supported in part by the Natural Sciences and Engineering Research Council (NSERC) of Canada.

${ }^{\dagger}$ Department of Computer Science, University of Toronto; 10 King's College Rd, Toronto, ON, M5S 3G4, Canada; krj@cs.toronto.edu

${ }^{\ddagger}$ Algorithmics Inc.; 185 Spadina Ave, Toronto, ON, M5T 2C6, Canada; alex.kreinin@algorithmics.com

$\S$ Department of Computer Science, University of Toronto; 10 King's College Rd, Toronto, ON, M5S 3G4, Canada; zhangw@cs.toronto.edu
} 
the link between analytical methods and the martingale approach is considered, the paper [4] discusses computational aspects of the problem, Taylor expansions of the probability distribution of $\tau$ are considered in [6].

Models using the first hitting time distribution find extensive application in the areas of portfolio credit risk modeling [8], [9] and pricing of credit derivatives [1], [5]. Here the process represents, so-called, distance to default of an obligor (see [1]), the first hitting time represents a default event and the boundary separates the healthy states of the obligor from the default state. For this reason, the boundary, $b(t)$, is often called the default boundary in the applied literature.

Calibration of the default model in the credit risk context leads to the inverse problem for the process $X_{t}$ :

Given a process, $X_{t}$, and the distribution, $F(t)$, find a boundary, $b(t)$, such that $\mathbb{P}(\tau \leq t)=F(t)$.

This problem was considered in [9] for the case $X_{t}$ is a Brownian random walk ${ }^{1}$. A detailed analysis of the inverse problem in the discrete time setting is given in [8] as well as a solution based on the Monte Carlo method. This approach is applicable to a much more general class of processes $X_{t}$, not just Brownian random walks.

Existence of the solution to the continuous-time inverse problem is analyzed in [2]. In [13], an integral equation for the boundary is derived when $X_{t}$ is a Brownian motion.

Unfortunately, this problem appears to be very difficult: an analytical solution is known in a few cases only. In this paper, we study a modified inverse problem. We consider the process $X_{t}=a+W_{t}$ with a random initial position, $X_{0}=a$, where $W_{t}$ is a standard Wiener process. The boundary, $b(t)=\mu t$, is linear.

We assume that $\mu \geq 0$ and $a$ is a non-negative random variable, $\mathbb{P}(a \geq 0)=1$. The first hitting time is denoted by $\tau^{(\mu)}$. The modified inverse problem is formulated as follows:

Find a distribution of the random variable, $a$,

$$
\text { such that } \mathbb{P}\left(\tau^{(\mu)} \leq t\right)=F(t) \text {. }
$$

In particular, we are interested in the solution of Problem (1) for $F(t)$ belonging to the class of Gamma distributions with the probability density function

$$
p_{\gamma}(t)=\lambda \cdot \frac{(\lambda t)^{\gamma-1}}{\Gamma(\gamma)} \cdot \exp (-\lambda t), \quad \gamma>0, \lambda>0 .
$$

The randomization of the initial state approach has been applied to credit risk modeling by Duffie and Lando [3]. The randomization of the initial state of the process $X_{t}$ allows us to find an analytically tractable solution for a large class of distributions, $F(t)$, without changing the covariance structure of the process. The idea behind our solution is as follows: given $X_{0}=a$, the conditional density function of $\tau^{(\mu)}, f_{a}(t)$, and the conditional distribution function,

$$
F_{a}(t)=\mathbb{P}\left(\tau \leq t \mid X_{0}=a\right),
$$

\footnotetext{
${ }^{1} X_{t}$ is a discrete time process with Gaussian increments.
} 
are available in closed form. Let $g(a)$ be the density function of the initial state, $X_{0}=a$. Then, the distribution function, $F(t)$, of the first hitting time satisfies

$$
F(t)=\int_{0}^{\infty} F_{a}(t) g(a) \mathrm{d} a .
$$

Equation (2) shows that the random variable $\tau^{(\mu)}$ is the mixture of the random variables, $\tau_{a}^{(\mu)}$, representing the first hitting time for the process $X_{t}=W_{t}-\mu t$ and the constant boundary, $b(t)=-a$. The density function of $\tau_{a}^{(\mu)}$ has a simple analytical form. From Equation (2) we find the Laplace transform, $\hat{g}(s)$, of the density function, $g$, and derive conditions on the parameter $\mu$ providing existence of the solution.

The rest of the paper is organized as follows. In Section 2 we review some of the existing results on the first hitting time distribution of a Wiener process. Section 3 presents our solution to the inverse Problem (1). Section 4 discusses existence of the density function $g(a)$. Section 5 concludes the paper.

\section{First hitting time for a Wiener process}

We start this section with a brief review of the properties of the first hitting time of a Wiener process $W_{t}$ with constant and linear boundaries [10].

Consider, first, the constant boundary $b(t)=-a, a>0$. In this case, $\mu=0$. Using the reflection principle,

$$
\mathbb{P}\left\{\tau_{a}^{(0)} \leq t\right\}=2 \mathbb{P}\left\{W_{t} \leq-a\right\}=2 \Phi\left(\frac{-a}{\sqrt{t}}\right),
$$

where $\Phi(x)$ is the standard normal cumulative distribution function.

The probability density function of the first hitting time satisfies the relation

$$
f_{a}(t)=\frac{a}{\sqrt{2 \pi t^{3}}} \exp \left(-\frac{a^{2}}{2 t}\right)
$$

In the case of a constant boundary (i.e., $\mu=0$ ), the random variable, $\tau_{a}^{(0)}$, is finite almost surely but $\mathbb{E} \tau_{a}^{(0)}=\infty$. If we take a mixture of random variables, $\tau_{a}^{(0)}$, with a mixing density, $g$, the resulting random variable, $\tau^{(0)}$, will also have infinite expectation. In this case it is not possible to match a class of distributions, $F(t)$, with a finite first moment.

Let us now consider the first hitting time, $\tau_{a}^{(\mu)}$, of a Brownian motion with drift, $X_{t}=$ $W_{t}-\mu t$, and a constant boundary $-a$. Obviously, the first hitting time for $X_{t}$ coincides with the first hitting time of $W_{t}$ and the boundary $b_{\mu}(t)=\mu t-a$. Using the Girsanov theorem we find ${ }^{2}$

$$
\mathbb{P}\left(\tau_{a}^{(\mu)} \leq t\right)=\int_{0}^{t} \frac{a}{\sqrt{2 \pi s^{3}}} \exp \left(-\frac{(a-\mu s)^{2}}{2 s}\right) \mathrm{d} s .
$$

Therefore, given a value of $a$, the conditional density function, $f_{a}(t)$, for the first hitting time of $W_{t}-\mu t$ with the boundary $-a$ (or equivalently for the first hitting time of $W_{t}$ with the

\footnotetext{
${ }^{2}$ The details of the derivation can be found in [10].
} 
boundary $\mu t-a)$ and the conditional distribution function are given by

$$
\begin{aligned}
& f_{a}(t)=\frac{a}{\sqrt{2 \pi t^{3}}} \exp \left(-\frac{(a-\mu t)^{2}}{2 t}\right) \\
& F_{a}(t)=\Phi\left(\frac{-a+\mu t}{\sqrt{t}}\right)+e^{2 \mu a} \Phi\left(\frac{-a-\mu t}{\sqrt{t}}\right) .
\end{aligned}
$$

From (6) it follows that

$$
\mathbb{P}\left(\tau_{a}^{(\mu)}<\infty\right)= \begin{cases}1, & \text { if } \mu \geq 0, \\ e^{2 \mu a}, & \text { otherwise }\end{cases}
$$

Thus, if $\mu>0$, then the first hitting time in our problem is finite almost surely. The moment generating function, $G_{a}(s)=\mathbb{E}\left[e^{s \tau_{a}^{(\mu)}}\right]$, is (see [10])

$$
G_{a}(s)=\int_{0}^{\infty} e^{s t} f_{a}(t) \mathrm{d} t=e^{\left(\mu-\sqrt{\mu^{2}-2 s}\right) a} .
$$

If $\mu>0$, the expected value of the first hitting time, $\tau_{a}^{(\mu)}$, is finite:

$$
\mathbb{E}\left[\tau_{a}^{(\mu)}\right]=\frac{a}{\mu}
$$

Therefore

$$
\mathbb{E}\left[\tau^{(\mu)}\right]=\frac{\mathbb{E}[a]}{\mu}
$$

\section{Density function $g(a)$}

Now we can find the relation between the distribution function, $F(t)$, and the density function, $g(\cdot)$, of the initial distribution of the process $X_{t}$.

Proposition 1. Suppose that the random variable, $\tau^{(\mu)}$, has an absolutely continuous distribution function, $F(t)$, with the density function $f(t)$. Denote the Laplace transform of $f$ by

$$
\hat{f}(s)=\int_{0}^{\infty} e^{-s t} f(t) \mathrm{d} t, \quad s \geq 0 .
$$

If a density function of the initial state, $X_{0}=a$, exists, then the Laplace transform

$$
\hat{g}(s)=\int_{0}^{\infty} e^{-s t} g(t) \mathrm{d} t
$$

satisfies the equation

$$
\hat{g}(s)=\hat{f}\left(\frac{s(s+2 \mu)}{2}\right)
$$

REmark. The function $\hat{g}(s)$ defined by Equation (9) may not be a Laplace transform of a density function of a random variable. In this case, a solution to Problem (1) does not exist. For this reason, we formulate Proposition 1 in a conditional form. 
Proof. The density function, $f(t)$, of the random variable $\tau^{(\mu)}$ satisfies the equation

$$
f(t)=\int_{0}^{\infty} f_{a}(t) \cdot g(a) \mathrm{d} a
$$

Applying the Laplace transform to this equation, we find

$$
\hat{f}(s)=\int_{0}^{\infty} G_{a}(-s) g(a) \mathrm{d} a .
$$

Since $G_{a}(-s)=\exp \left(a \mu-a \sqrt{\mu^{2}+2 s}\right)$, we obtain

$$
\hat{f}(s)=\hat{g}\left(\sqrt{\mu^{2}+2 s}-\mu\right) .
$$

The latter equation is equivalent to (9).

Let us now find the density $g(a)$ if the first hitting time distribution is the Gamma distribution. Let $f(t)=p_{\gamma}(t)$, for some $\gamma>0$. Then we have

$$
\hat{f}(s)=\frac{\lambda^{\gamma}}{(\lambda+s)^{\gamma}}
$$

From Equation (9) we obtain

$$
\hat{g}(s)=\frac{(2 \lambda)^{\gamma}}{\left((s+\mu)^{2}-\left(\mu^{2}-2 \lambda\right)\right)^{\gamma}} .
$$

Rearranging (11), we find

$$
\hat{g}(s)=\frac{\left(\mu+\sqrt{\mu^{2}-2 \lambda}\right)^{\gamma}}{\left(s+\mu+\sqrt{\mu^{2}-2 \lambda}\right)^{\gamma}} \cdot \frac{\left(\mu-\sqrt{\mu^{2}-2 \lambda}\right)^{\gamma}}{\left(s+\mu-\sqrt{\mu^{2}-2 \lambda}\right)^{\gamma}} .
$$

The latter representation of the Laplace transform, $\hat{g}(s)$, corresponds to the sum of two independent random variables, $\xi_{1}+\xi_{2}$, having Gamma distributions with a common shape parameter $\gamma: \xi_{1} \sim \Gamma\left(\gamma, \lambda_{1}\right)$ and $\xi_{2} \sim \Gamma\left(\gamma, \lambda_{2}\right)$, where

$$
\lambda_{1}=\mu-\sqrt{\mu^{2}-2 \lambda}, \quad \lambda_{2}=\mu+\sqrt{\mu^{2}-2 \lambda} .
$$

From (11) it follows that $\mu$ must satisfy the inequality

$$
\mu \geq \sqrt{2 \lambda}
$$

otherwise the solution of Problem (1) does not exist. Indeed, if $\mu<\sqrt{2 \lambda}$, the function, $g(a)$, corresponding to $\hat{g}(s)$, takes negative values. Thus, we proved

Proposition 2. Consider the first hitting time of the process $X_{t}=a+W_{t}$ and the linear boundary $b(t)=\mu t$. If the distribution $F(t)$ belongs to the class of Gamma distributions with the rate parameter $\lambda$ satisfying Inequality (12), then Problem (1) has a solution and the Laplace transform of the distribution of the initial state $X_{0}=a$ is given by (11). If Inequality (12) is not satisfied, then the solution does not exist. 


\section{Existence of a solution}

In the previous section, we reduced the inverse problem to the solution of the integral equation for the density function, $g(\cdot)$,

$$
\hat{f}(s)=\int_{0}^{\infty} G_{a}(-s) \cdot g(a) \mathrm{d} a, \quad s>0
$$

where $G_{a}(s)$ is defined by Equation $(8)$ and $\hat{f}(s)$ is the Laplace transform of a given density function $f(t)$. In the case $f(t)=p_{\gamma}(t)$ we found the solution to the problem defined by (8) if the parameter $\mu$ satisfies (12).

Now we would like to find general conditions on the Laplace transform, $\hat{f}(s)$, providing existence of the density function, $g(a)$, satisfying the integral equation (13). The following proposition gives a sufficient condition for existence of the density $g(a)$.

Proposition 3. Fix the boundary $b(t)=\mu t$. Consider a non-negative random variable, $\tau$. Let $\mathfrak{R}$ denote the class of functions $\hat{f}(s)=\mathbb{E}\left[e^{-s \tau}\right]$ of the argument s represented in the form

$$
\hat{f}(s)=\sum_{k=1}^{K} \frac{\alpha_{k}}{\left(s+\lambda_{k}\right)^{l_{k}}}, \quad l_{k} \in \mathbb{R}_{+}, \quad \lambda_{k}, \alpha_{k}>0 .
$$

Then there exists $\mu_{*}>0$ such that for all $\mu \geq \mu_{*}$ the integral equation (13) has a solution, $g(a)$, satisfying the conditions

$$
g(a) \geq 0, \quad \int_{0}^{\infty} g(a) \mathrm{d} a=1
$$

Proof. We start the proof of Proposition 3 with the following remark. Let $\vec{p}=\left(p_{1}, p_{2}, \ldots, p_{K}\right)$ be a finite probability distribution. Therefore

$$
\sum_{k=1}^{K} p_{k}=1, \quad p_{k} \geq 0 \quad \text { for } k=1,2, \ldots, K .
$$

If each density function, $g_{k}(a),(k=1,2, \ldots, K)$, solves Problem (1) for each associated density $f_{k}(t)$ and the linear boundary $b(t)=\mu t$, then the convex combination

$$
g(a)=\sum_{k=1}^{K} p_{k} g_{k}(a)
$$

solves Problem (1) for the density

$$
f(t)=\sum_{k=1}^{K} p_{k} f_{k}(t)
$$

Let us now consider a density function $f(t)$ such that $\hat{f}(s) \in \mathfrak{R}$. Since $\hat{f}(0)=1$ and $\alpha_{k}>0$, $\lambda_{k}>0$, we obtain

$$
\sum_{k=1}^{K} \frac{\alpha_{k}}{\lambda_{k}^{l_{k}}}=1
$$


Denote $p_{k}=\frac{\alpha_{k}}{\lambda_{k}^{l_{k}}}$. Obviously, $p_{k}>0$ and $\sum_{k=1}^{K} p_{k}=1$. Then the function $\hat{f}(s)$ defined by (14) can be represented as a convex combination

$$
\hat{f}(s)=\sum_{k=1}^{K} p_{k} \hat{f}_{k}(s),
$$

where

$$
\hat{f}_{k}(s)=\frac{\lambda_{k}^{l_{k}}}{\left(s+\lambda_{k}\right)^{l_{k}}}
$$

The Laplace transform, $\hat{f}_{k}(s)$, corresponds to a random variable, $\tau_{k} \sim \Gamma\left(l_{k}, \lambda_{k}\right)$, having a Gamma distribution. Denote

$$
\mu_{*}=\max _{1 \leq k \leq K} \sqrt{2 \lambda_{k}}
$$

Then, by Proposition 2, for $\mu>\mu_{*}$ and for each $k=1,2, \ldots, K$ there exists $g_{k}(a)$ solving Problem (1) for the density $f_{k}(t)$. Consequently, the density

$$
g(a)=\sum_{k=1}^{K} p_{k} g_{k}(a)
$$

solves Problem (1) for the probability density function

$$
f(t)=\sum_{k=1}^{K} p_{k} \lambda_{k} \exp \left(-\lambda_{k} t\right) \frac{\left(\lambda_{k} t\right)^{l_{k}}}{\Gamma\left(l_{k}\right)} .
$$

The Proposition is, thus, proved.

The convex closure of the class $\mathfrak{R}$ of rational functions $\hat{f}(s)$ contains Laplace transforms of all non-negative random variables. That is, for any random variable $\xi \geq 0$, there exists a sequence of random variables $\xi_{n} \geq 0$ such that

$$
\lim _{n \rightarrow \infty} \mathbb{E}\left[\exp \left(-s \xi_{n}\right)\right]=\mathbb{E}[\exp (-s \xi)] \quad \forall s \geq 0
$$

and $\mathbb{E}\left[\exp \left(-s \xi_{n}\right)\right] \in \mathfrak{R}$. However, this property does not allow us to conclude that Problem (1) can be solved for an arbitrary distribution $F(t)$. Consider, for instance, a degenerate random variable, $\tau$, such that $\mathbb{P}(\tau=c)=1$, where $c>0$ is a constant. In this case

$$
\mathbb{E}[\exp (-s \tau)]=e^{-s c}
$$

Let $\lambda_{k}=k \cdot c^{-1}$ and $l_{k}=k,(k=1,2, \ldots)$. Consider a sequence of random variables, $\tau_{k}$, having Gamma distributions, $\tau_{k} \sim \Gamma\left(\lambda_{k}, l_{k}\right),(k=1,2, \ldots)$. Then we have

$$
\mathbb{E}\left[\exp \left(-s \tau_{k}\right)\right]=\frac{\lambda_{k}^{l_{k}}}{\left(\lambda_{k}+s\right)^{l_{k}}}=\frac{1}{\left(1+\frac{s c}{k}\right)^{k}},
$$

and

$$
\lim _{k \rightarrow \infty} \mathbb{E}\left[\exp \left(-s \tau_{k}\right)\right]=e^{-s c}, \quad s \geq 0,
$$

but there is no finite $\mu$, satisfying the inequality $\mu>\sqrt{2 \lambda_{k}}$ for all $k$. Thus Problem (1) does not have a solution for any $\mu$ in this case. 
For the same reason, there is no solution to Problem (1) if the function $F(t)$ is discontinuous. If we impose the condition: there exists $\lambda_{*}>0$ such that

$$
\lambda_{k} \leq \lambda_{*} \quad \text { for all } k=1,2, \ldots
$$

then for any $\mu \geq \sqrt{2 \lambda_{*}}$ there exists a solution, $g_{k}(a)$, of Problem (1) given the density $f_{k}(t)$, and the mixture

$$
g(a)=\sum_{k \geq 0} p_{k} g_{k}(a)
$$

solves Problem (1) for

$$
f(t)=\sum_{k \geq 0} p_{k} f_{k}(t)
$$

The class of distributions satisfying condition (16) has a positive density, $f(t)$, for all $t>0$.

Proposition 3 defines a class $\mathfrak{R}$ for which a density $g(a)$ exists for $\mu>0$. As mentioned above, if $\mu=0$, it is impossible to match a class of distributions with a finite first moment, such as the class $\mathfrak{R}$. The following proposition gives a sufficient condition for the existence of the density for the case $\mu=0$.

Proposition 4. Fix the boundary $b(t)=0$. Consider a non-negative random variable, $\tau$. Let $\mathfrak{R}_{0}$ denote the class of functions $\hat{f}(s)=\mathbb{E}\left[e^{-s \tau}\right]$ of the argument s represented in the form

$$
\hat{f}(s)=\sum_{k=1}^{K} \frac{\alpha_{k}}{\left(\sqrt{2 s}+\lambda_{k}\right)^{l_{k}}}, \quad l_{k} \in \mathbb{R}_{+}, \quad \lambda_{k}, \alpha_{k}>0 .
$$

Then the integral equation (13) has a solution, $g(a)$, satisfying the conditions

$$
g(a) \geq 0, \quad \int_{0}^{\infty} g(a) \mathrm{d} a=1 .
$$

Proof. Similar to the proof of Proposition 3, the function $\hat{f}(s)$ defined in (17) can be represented as a convex combination

$$
\hat{f}(s)=\sum_{k=1}^{K} p_{k} \hat{f}_{k}(s)
$$

where

$$
p_{k}=\frac{\alpha_{k}}{\lambda_{k}^{l_{k}}} \quad \text { and } \quad \hat{f}_{k}(s)=\frac{\lambda_{k}^{l_{k}}}{\left(\sqrt{2 s}+\lambda_{k}\right)^{l_{k}}} .
$$

From (9) of Proposition 1, we have

$$
\hat{g}_{k}(s)=\hat{f}_{k}\left(\frac{s^{2}}{2}\right)=\frac{\lambda_{k}^{l_{k}}}{\left(s+\lambda_{k}\right)^{l_{k}}}
$$

which corresponds to a Gamma distribution

$$
g_{k}(a)=\lambda_{k} \exp \left(-\lambda_{k} a\right) \frac{\left(\lambda_{k} a\right)^{l_{k}}}{\Gamma\left(l_{k}\right)} .
$$


Then the density

$$
g(a)=\sum_{k=1}^{K} p_{k} g_{k}(a)
$$

solves the Problem (1) with $\mu=0$ for the density function $f(t)$ with Laplace transform $\hat{f}(s) \in \mathfrak{R}_{0}$. Therefore, the Proposition is proved.

\section{Conclusion}

In this paper we found a sufficient condition for existence of a solution of the modified inverse problem for the first hitting time distribution. We proved that the problem has a closed form solution in the class of distributions satisfying inequality (16) for the partial fractions in the representation of the Laplace transform, if the linear boundary, $b(t)=\mu t$, satisfies the condition $\mu>\sqrt{2 \lambda_{*}}$. The problem also has a closed form solution in the class of distributions with Laplace transform (17), if $\mu=0$. The distribution of the initial state of the process can be described in both cases as a mixture of sums of Gamma-distributed random variables.

It would be interesting to find also necessary conditions for existence of the solution of the modified inverse problem. The randomization idea can also be applied to solve some special cases of the Skorokhod stopping problem, which is to find $\tau$ such that the distribution of $X_{\tau}$ is given.

\section{References}

[1] M. Avellaneda and J. Zhu. Distance to default. Risk, 14(12):125-129, 2001.

[2] J. Chadam, L. Cheng, X. Chen, and D. Saunders. Analysis of an inverse first passage problem from risk management. SIAM Journal on Mathematical Analysis, 38(3), 845$873,2006$.

[3] D. Duffie and D. Lando. Term structures of credit spreads with incomplete accounting information. Econometrica, 69(3), 633-664, 2001.

[4] J. Durbin. The first passage density of the Brownian motion process to a curved boundary. Journal of Applied Probability, 29, 291-304, 1992.

[5] J. Hull and A. White. Valuing credit default swaps II: modeling default correlations. Journal of Derivatives, 8(3), 2001.

[6] D. G. Hobson, D. Williams, and A. Wood. Taylor expansions of curve-crossing probabilities. Bernoulli, 5(5), 779-795, 1999.

[7] T. R. Hurd. Credit risk modeling using time-changed Brownian motion. McMaster University, Working paper, 2007.

[8] I. Iscoe and A. Kreinin. Default boundary problem. Technical report, Algorithmics Inc., 1999. 
[9] I. Iscoe, A. Kreinin, and D. Rosen. Integrated market and credit risk portfolio model. Algorithmics Research Quarterly, 2(3), 21-38, 1999.

[10] I. Karatzas and S. E. Shreve. Brownian Motion and Stochastic Calculus. SpringerVerlag, New York, 2005.

[11] A. Ya. Khintchine. Asymptotische Gesetze der Wahrscheinlichkeits Rechnuung. Springer, Berlin, 1933.

[12] H. R. Lerche. Boundary crossing of Brownian motion. Lecture Notes in Statistics 40. Springer-Verlag, New York, 1986.

[13] G. Peskir. On integral equation arising in the first-passage problem for Brownian motion. Journal Integral Equations and Applications, 14, 397-423, 2002. 